Xil International Symposium on Radiation from Relativistic Electrons in Periodic Structures - RREPS-17

18-22 September, 2017

DESY, Hamburg, Germany

\title{
Experimental station with continuous electron beam for investigation of various mechanisms of EM radiation generation
}

\author{
R.M. Nazhmudinov, ${ }^{a, c, 1}$ P. Karataev, ${ }^{b}$ A. Kubankin,,${ }^{a, c}$ K. Lekomtsev, ${ }^{b}$ A. Potylitsyn ${ }^{d}$ \\ and A. Vukolov ${ }^{d}$ \\ ${ }^{a}$ Belgorod National Research University, \\ 85, Pobedy St., Belgorod, 308015, Russian Federation \\ ${ }^{b}$ John Adams Institute at Royal Holloway University of London, \\ Egham, Surrey TW20 OEX, United Kingdom \\ ${ }^{c}$ P.N. Lebedev Physical Institute of the Russian Academy of Sciences, \\ 53, Leninskiy Prospekt, Moscow, 119991 GSP-1, Russian Federation \\ ${ }^{d}$ Tomsk Polytechnic University, \\ 2, Pr. Lenina, Tomsk, 634050, Russian Federation \\ E-mail: Nazhmudinov@bsu.edu.ru
}

AвstRact: In this report we describe a new facility designed, manufactured and commissioned at Belgorod National Research University. It is a compact and cost-efficient low energy continuous electron beam installation developed to underpin research and education foundation of the University group. We present the measurements of the beam size, emittance, position and trajectory. A $\mathrm{THz}$ detection beam line has also been constructed and tested. The status of the $\mathrm{THz}$ radiation generation programme, future upgrades and experimental plans have been described.

KeYwords: Accelerator Applications; Beam-line instrumentation (beam position and profile monitors; beam-intensity monitors; bunch length monitors); Photon detectors for UV, visible and IR photons (gas)

${ }^{1}$ Corresponding author. 


\section{Contents}

1 Introduction 1

2 Continuous electron beam facility 2

2.1 DC gun and beam transport 2

2.2 Experimental vacuum vessel 3

2.3 Electron beam diagnostics 4

3 Commissioning of the THz radiation measurement line 5

4 Conclusion $\quad 7$

\section{Introduction}

Electromagnetic radiation sources based on particle accelerators have been a primary area of research in accelerator physics community over the past few decades (see, for instance, [1, 2]). This direction requires high gradient and efficient accelerating structures. However the technology has nearly reached its limits partially due to breakdown problems at high fields, partially due to the lack of affordable higher power sources. Due to complicated particle beam dynamics long accelerators experience additional limitations on high quality beam parameters [3, 4].

Nowadays accelerator community is seeking for novel ways to generate high field accelerating gradients in compact structures. One of the approaches is to use dielectric micro-channels [5, 6] with or without internal structure to accelerate particle beams. These techniques require high power $\mathrm{THz}$ radiation sources to drive the accelerating structure. On the other hand, compact high power $\mathrm{THz}$ sources are needed to underpin research in such disciplines as physics, chemistry, and biology due to the fact that many materials leave spectral signatures at $\mathrm{THz}$ frequencies enabling investigations of their structural characteristics.

THz lasers [7] have very low average power. At the moment there are two schemes for generating intense EM radiation beams in sub-THz frequency range: an orotron and a gyrotron. Both schemes utilize high peak power pulsed electron beam. In orotrons [8] the radiation is generated by intense electron pulse passing through an open resonator with integrated metallic grating. The grating defines the emitted radiation wavelength. In a gyrotron [9] EM radiation is emitted via stimulation of the cyclotron resonance in a strong magnetic field. Despite of such significant difference in technology and the mechanisms, both methods experience serious difficulties at radiation frequencies above $1 \mathrm{THz}$, where the generation efficiency drops down drastically.

In Radiation Physics Laboratory of Belgorod National Research University we have launched a new programme on development of compact cost-efficient continuous electron beam experimental assembly consisting of an electron gun with a heated tungsten filament, beam transport (a condenser lens and two pairs of bending magnets) and an experimental area. The particle beam has the 
following beam parameters: energy up to $100 \mathrm{keV}$, DC current of up to $500 \mu \mathrm{A}$, beam size of about $1-1.5 \mathrm{~mm}$, and angular divergence of less than $0.5^{\circ}$. The purpose is to develop a continuous electron beam system affordable by a small industrial team or a university group enabling a broad range of fundamental and applied research.

An experimental programme on investigation of various mechanisms for generating intense $\mathrm{THz}$ radiation beams using a low energy high current $\mathrm{DC}$ electron beams has been initiated. We aim in investigating the conditions and limitations of generating EM radiation in the frequency range from 1 to $10 \mathrm{THz}$. The installation can be used to generate intense UV and soft X-rays via direct interaction of electrons with amorphous or crystalline materials as an alternative to largescale central light source facilities. The experimental area is equipped with diagnostics equipment capable of measuring beam size, emittance, position, direction of propagation, and the beam current.

In this paper we present the assembly prototype including a DC gun, vacuum hardware, diagnostics equipment, radiation measurement equipment and data acquisition system. All major parameters: beam current, beam size versus longitudinal position, and beam emittance have been characterised. Advanced plan for the future engineering and experimental work is summarized in the conclusion.

\section{Continuous electron beam facility}

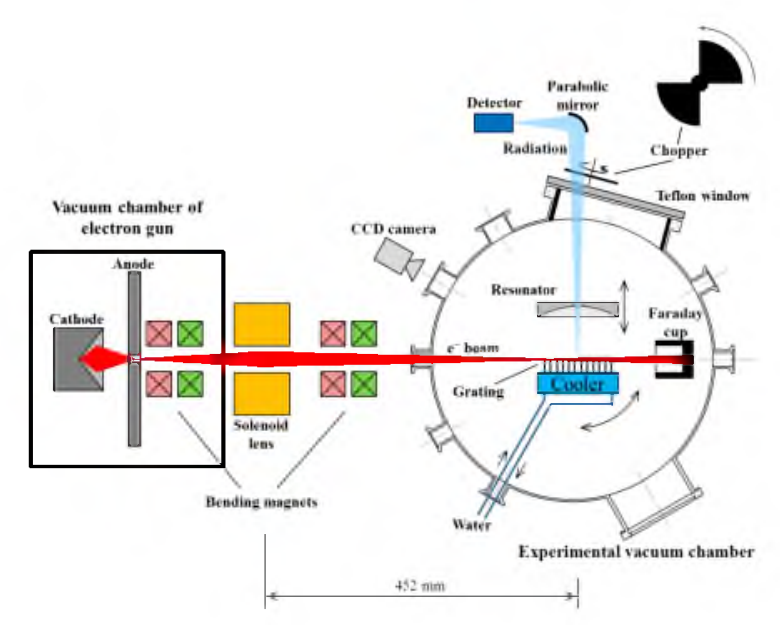

Figure 1. A complete layout of the facility.

The complete experimental layout of the continuous electron beam facility is schematically illustrated in figure 1. The facility consists of the DC electron gun, beam transport system, experimental vacuum vessel and a set of diagnostics equipment.

\subsection{DC gun and beam transport}

The electron gun and the potential distribution in it are shown in figure 2. It produces an electron beam with energy variable in the range from $10-100 \mathrm{keV}$. The beam current of up to $500 \mu \mathrm{A}$ can be achieved. The electron gun has a V-shaped tungsten filament (produced by Kimball Physics Inc.) as a thermionic emitter. A high negative potential is applied to the cathode. An example 
of electromagnetic simulations of the applied potential is demonstrated in figure 2 (right). The working pressure in the gun chamber is $10^{-7}$ Torr.

The initial focusing of the beam is enabled due to a conical shape of the cathode. As is seen from figure 2 (left) such cathode shape enables to achieve flat capture field and preserve the emittance dilution. The anode is a diaphragm with an aperture diameter of $0.7 \mathrm{~mm}$ with the ground potential. The shape of the beam is controlled by a solenoid condenser lens. The electron beam trajectory is controlled by two pairs of dipole magnetic deflectors. To protect against the X-rays, the experimental vacuum chamber and the electron gun are surrounded by lead shielding.
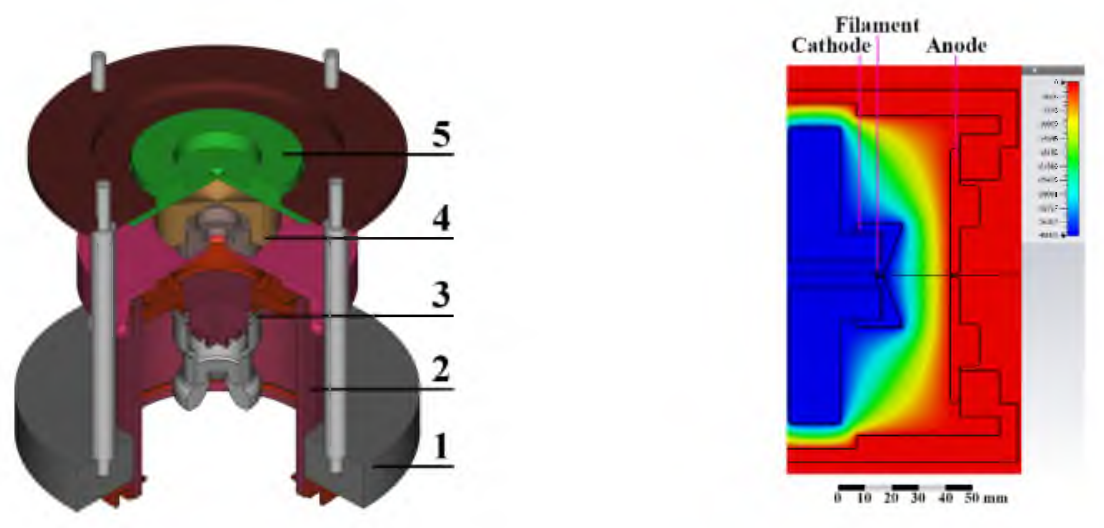

Figure 2. 3D image of the electron gun (left) and example of potential distribution (right): $I$ - mounting flange, 2 - insulator, 3 - HV connector, 4 - cathode (HV electrode), 5 - anode.

\subsection{Experimental vacuum vessel}

The experimental vacuum vessel is schematically demonstrated in figure 1. It is a cylindrical vacuum chamber of $400 \mathrm{~mm}$ in diameter. It has several small feedthrough vacuum ports for electrical connections, externally controlled vacuum actuators and viewport observations. A large scale view port is typically used for radiation measurements.

The electron beam is steered into the experimental area with a set of dipole magnets. A target for far-infrared radiation generation is installed on a two-axes in-vacuum goniometer which enables us to control the position and the angle of the target relative to electron beam trajectory with accuracy of the order of $2.5 \mu \mathrm{m}$ and $0.01^{\circ}$ respectively. The radiation propagates to the $\mathrm{THz}$ measurement beam line through $8 \mathrm{~mm}$ thick Teflon viewport (transparent for far-infrared and $\mathrm{THz}$ frequencies).

The working pressure in the experimental vessel it is $10^{-5}$ Torr. The experimental vacuum vessel is separated from the gun chamber by a slide gate valve so that we can break the vacuum and modify experimental setup while keeping the gun chamber evacuated. Each chamber has a twostage vacuum system, including turbomolecular and spiral pumps. The pressure in the chambers is measured by combined vacuum gauges with inverse magnetron sensors for high vacuum and Pirani sensors for low vacuum. 


\subsection{Electron beam diagnostics}

The beam diagnostics is an integral part of the assembly. It can measure a variety of the beam parameters including transverse beam size, emittance, position, direction of propagation, and beam charge. These parameters are essential for result interpretation of any physics experiment.

The electron beam current monitored by a Faraday cup is installed at the end of the beam line. It is also used to safely terminate the beam after use.

The shape of the electron beam is measured by a

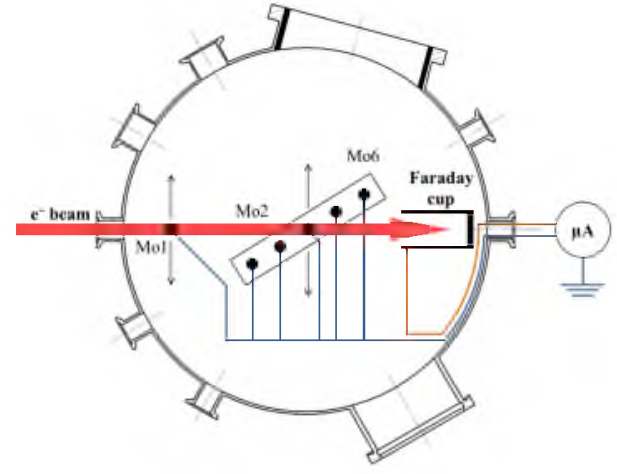

Figure 3. Location of wire scanners. transverse multi-wire scanner, which includes six molybdenum $0.1 \mathrm{~mm}$ thick wire sensors. $\mathrm{Mol}$ is mounted on a separate translation stage at the entrance to the experimental area. Mo2. . Mo6 are mounted on a single frame. The frame itself is attached to the target manipulation system as shown in figure 3. The particle beam induces current in the wires, which is registered by the data acquisition system. Thus, the scanner makes it possible to measure the spatial beam profiles at six different positions along the beam. The accuracy of the wire motion is smaller than $2.5 \mu \mathrm{m}$. To avoid the effect of the multiple scattering in the upstream wires on the measurement done by the downstream wires and to be able to acquire the signal from all wires by the same digitizer, the wire assembly is slightly tilted with respect to the beam trajectory, so that the beam interacts with each wire independently. Since all wires move simultaneously it reduces the measurement time. Typical electron beam profiles of $45 \mathrm{keV}$ electron beam are shown in figure 4 .
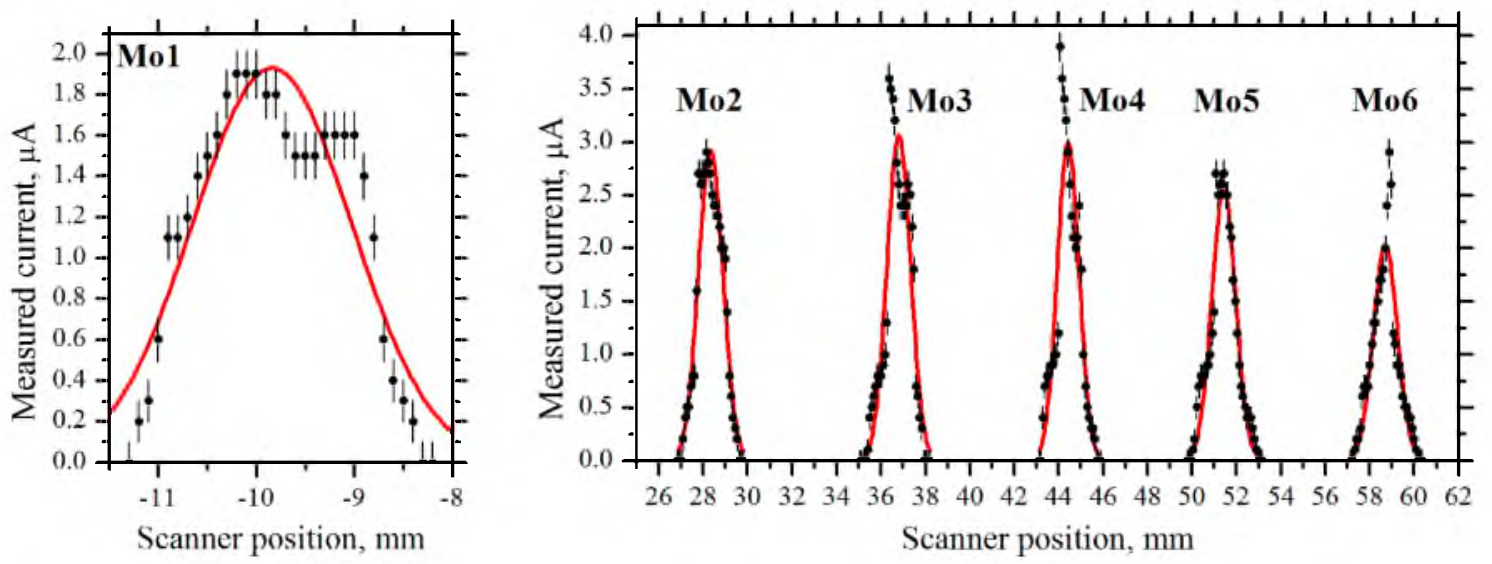

Figure 4. The electron beam profiles measured for energy of $45 \mathrm{keV}$ and current of $104 \mu \mathrm{A}$ : points are the measured values, the line is the Gaussian fit.

Figure 5 shows how the beam width changes along its trajectory. The measurements were performed for the beam current of $104 \mu \mathrm{A}$. However even if we reduce the beam current by a factor of 10 we see no difference in measured beam profiles. It means the space charge effect is really small because we have a continuous beam. This is one of the advantages of the continuous beam technology. 


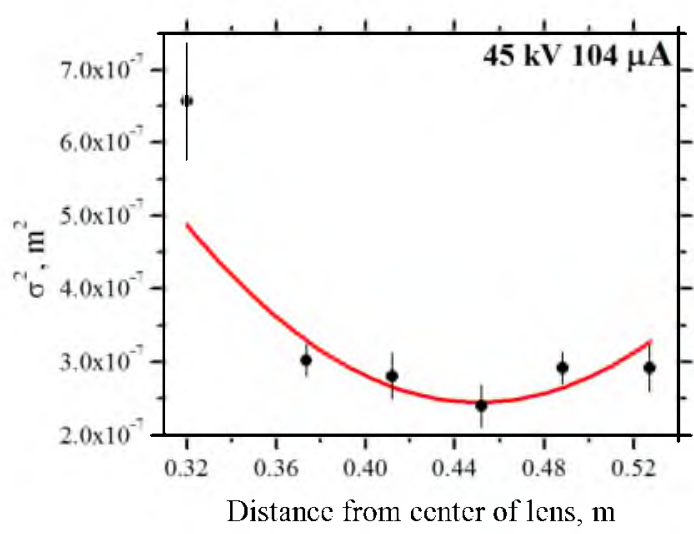

Figure 5. Beam size measured along the beam trajectory
The electron beam diameter near the target area is about $1.2 \mathrm{~mm}$ (at the distance $452 \mathrm{~mm}$ from the solenoid lens center). At $500 \mu \mathrm{A}$ we have $3 \cdot 10^{15}$ electrons $/ \mathrm{mm}^{2}$ per second which can potentially be increased by a factor of 10 . However in that case we need to use a special beam dump to avoid the hardware damage. For example. in [10] a pulsed beam of a lot more powerful facility has generated $10^{16}$ electrons $/ \mathrm{mm}^{2}$ per second. From the data shown in figure 5 the normalized emittance is extracted to be $\varepsilon_{n}=\beta \gamma \varepsilon=$ $0.8 \pm 0.5 \pi \mathrm{mm}$ mrad. where $\beta$ is the speed of particle in units of the speed of light, $\gamma$ is the charged particle Lorentz factor and $\varepsilon$ is the physical beam emittance. The uncertainty was extracted from the parabolic fit. It is rather high because the beam is not really Gaussian. However, even for the largest deviation, the emittance is still pretty small.

The position and trajectory of the electron beam is determined by the multi-wire centroid measurement.

For alignment and system tuning we installed a luminescent screen (which can be replaced by a conducting screen to generate transition radiation). The optical light is observed by a CCD camera. The screen is usually saturated because of very large number of electrons, however, it is a very useful tool for the beam line commissioning and the facility star-up tuning.

\section{Commissioning of the THz radiation measurement line}

The far-infrared radiation is extracted through a long Teflon window. The obscrvation angles from $60^{\circ}$ to $90^{\circ}$ relative to the electron beam trajectory can be achieved. The Golay detector Tydex GC-1P sensitive to the frequency range from $0.04-20 \mathrm{THz}$ was used in the experiment. The Golay Cell is a differential detector measuring the power variation. The output chopper modulates the output radiation so it can be detected. The chopper itself is a metal disk with two quadrants, which can rotate with a fixed frequency in the range from $5-20 \mathrm{~Hz}$. A parabolic mirror with effective area of $19.6 \mathrm{~cm}^{2}$ is used to concentrate the radiation power on the surface of the detector.

The data acquisition system is shown in figure 6. It includes the Golay Cell detector with Teflon cap (to cut the optical light background), a lock-in amplifier, an ADC module paired with a

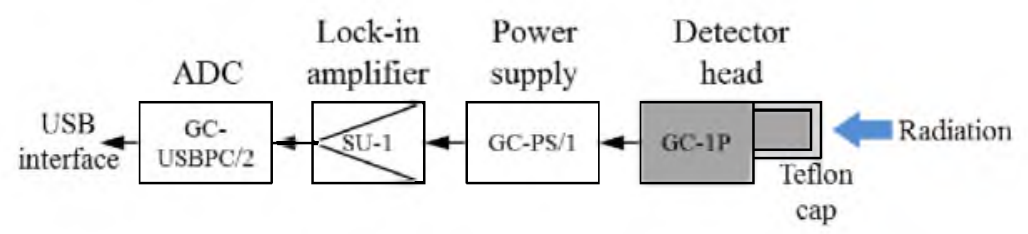

Figure 6. The data acquisition system (all units manufactured by Tydex). 
PC via a USB interface. The ADC module allows you to separate a useful signal with a specified modulation frequency from noise and to build the time dependence of its amplitude.

In order to estimate the characteristic relaxation time of our $\mathrm{THz}$ detector we measured the time dependence of the detector signal by blocking off (on) the light from the emission source (filament lamp). The dependence is shown in figure 7. The estimated relaxation time is $\tau_{0} \sim 4 \mathrm{~s}$.

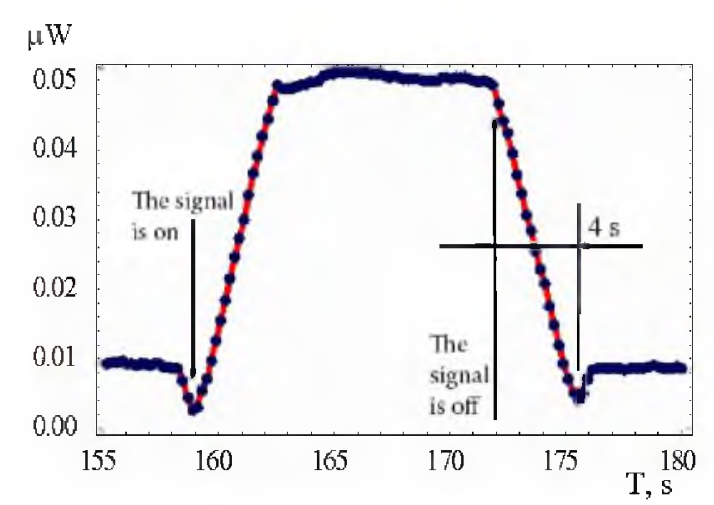

Figure 7. The relaxation time of the detecting system.

The far-infrared signal measurements were performed using the beam with parameters: $E_{\mathrm{e}}=$ $45 \mathrm{keV}$ and $I=300 \mu \mathrm{A}$.

We used two target types. The first one was a grating with period of $d=0.3 \mathrm{~mm}$ and groove depth of $t=3 \mathrm{~mm}$. The strips were made of permalloy. To reduce the radiation background generated by the grating material due to heating, it is cooled down by water. The number of periods was $N=316$. We expect the bandwidth of the first diffraction order defined as $1 / N=0.32 \%$ at $0.3 \mathrm{~mm}$ wavelength $(1 \mathrm{THz})$.

The impact parameter (the shortest distance between the beam and the grating) was $H<$ $1 \mathrm{~mm}$. The beam size was comparable to or larger than the impact parameter. A part of the beam was cleared by the target heating it up and generating the black body radiation. A resonator mirror was installed above the grating to return a part of the radiation back to the grating and stimulate the radiation production (standard orotron scheme, see [8]).

The second target was a $0.44 \mathrm{~mm}$ thick aluminium plate tilted relative to the beam at a shallow angle of about $1^{\circ}$. It produced transition radiation. At these energies transition radiation is nearly isotropic, therefore the target tilt angle is unimportant.
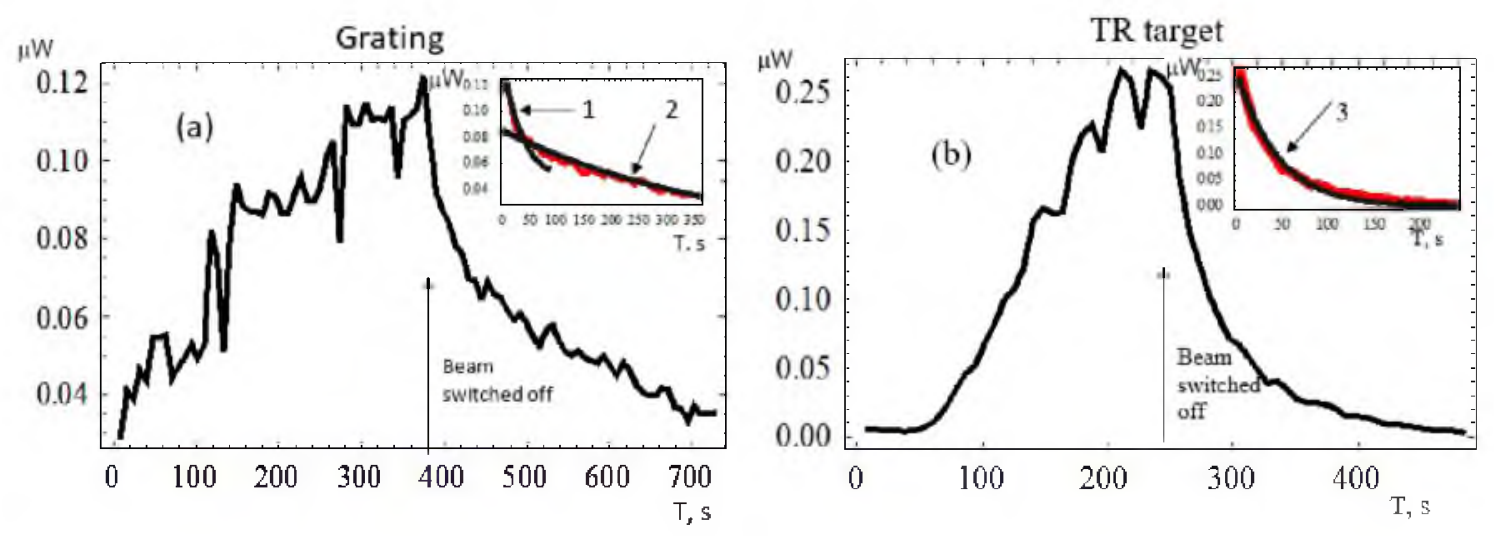

Figure 8. The time dependence of the observed radiation: a) diffraction grating, b) the aluminium plate.

The time dependences of the registered signals are shown in figure 8. From both targets we registered exponential decay as $\operatorname{Exp}\left(-t / t_{1}\right), t_{1}=33 \pm 2.6 \mathrm{~s}$ (curve 1 in the figure 8a, insert) and $\operatorname{Exp}\left(-t / t_{3}\right), t_{3}=43.5 \pm 1.4 \mathrm{~s}$ (curve 3 in the figure $8 \mathrm{~b}$, insert). Having in mind the slow decreasing 
of the signal from the grating (see range 2 in figure 8a, insert), for which the dependence is described by the exponent $\operatorname{Exp}\left(-t / t_{2}\right), t_{2}=400 \mathrm{~s}$ we can assume that the contribution to the detected signal from the polarization radiation mechanism is very weak. The signal is dominated by the black body radiation.

To roughly estimate the power emitted due to black body radiation generated from an Al plate and registered by our detection system we used the approach presented in [11]. The expected power level is $2 \mu \mathrm{W}$. According to the manufacturer data, our detector can easily detect this power level with high signal-to-noise ratio. That is what we observe in figure 8 .

\section{Conclusion}

In this report we have described a design of a new laboratory scale experimental facility designed, manufactured and commissioned at Belgorod National Research University. The facility consists of a continuous beam electron gun; beam transport system; vacuum chamber for generating electromagnetic radiation; beam charge, size and emittance, position and trajectory diagnostics equipment; $\mathrm{THz}$ measurement instrumentation and data-acquisition and analysis software. The main goal of this research is to develop a compact affordable electron source for generation of intense electromagnetic radiation beams. Our aim is to optimise conditions for producing radiation in the frequency range from $1-10 \mathrm{THz}$.

We have measured the continuous beam projected size to be $1.2 \mathrm{~mm}(\mathrm{rms})$ in the waist and emittance to be $0.8 \pm 0.5 \pi \mathrm{mm}$ mrad. The beam was stable and reproducible. This is an advantage of our system in comparison to similar facilities around the Globe. In [12] the authors estimated the emittance to be $0.4 \pi \mathrm{mm}$ mrad, however precise measurements are important for proper comparison with theoretical expectations.

We have demonstrated the status of the THz radiation generation programme. The interaction of the particle beam with an Al target and a grating demonstrated that the signal is dominated by the heat radiation emitted by the target material. Nevertheless, the measurements enable us to understand the $\mathrm{THz}$ detector response characteristics and radiation transport line alignment.

In the future we plan to add the longitudinal beam modulation to be able to reduce the heat background contamination and increase the radiation photon yield from a grating radiator. To be able to diagnose the longitudinal structure of the beam a transverse beam deflector will be employed.

\section{Acknowledgments}

The authors gratefully acknowledge the support of the program "NAUKA" of the Russian Ministry of Education and Science, grant TPU \#3.1903.2017, the European Union Horizon 2020 research and innovation programme under the Marie Sklodowska-Curie grant agreement No 655179.

\section{References}

[1] E. Chiadroni et al., The SPARC linear accelerator based terahertz source, Appl. Phys. Lett. 102 (2013) 094101.

[2] Diamond Light Source Ltd., http://www.diamond.ac.uk. 
[3] ATF2 collaboration, G.R. White et al., Experimental Validation of a Novel Compact Focusing Scheme for Future Energy-Frontier Linear Lepton Colliders, Phys. Rev. Lett. 112 (2014) 034802.

[4] D. Schulte, Emittance preservation in the main linac of CLIC, in proceedings of 6th European Particle Accelerator Conference, in Proceedings of the $6^{\text {th }}$ European Particle Accelerator Conference, Stockholm, Sweden, 22-26 Jun 1998, pp. 478-480 [CERN-PS-98-018].

[5] B.D. O'Shea, G. Andonian, S.K. Barber, K. L. Fitzmorris, S. Hakimi, J. Harrison et al., Observation of acceleration and deceleration in gigaelectron-volt-per-metre gradient dielectric wakefield accelerators, Nat. Commun. 7 (2016) 12763.

[6] K. Lekomtsev, A. Lyapin, S.T. Boogert, P.V. Karataev, J.S. Adams, A. Aryshev et al., Drive-witness Acceleration Scheme Based on Corrugated Dielectric Mm-scale Capillary, in Proceedings of the $8^{\text {th }}$ International Particle Accelerator Conference, Copenhagen, Denmark, 2017, pp. 3292-3295 [WEPVA018].

[7] A. Roggenbuck, H. Schmitz, A. Deninger, I. C. Mayorga, J. Hemberger, R. Güsten et al., Coherent broadband continuous-wave terahertz spectroscopy on solid-state samples, New J. Phys. 12 (2010) 043017.

[8] F.S. Rusin and G.D. Bogomolov, Generation of electromagnetic oscillation in an open resonator, $J$. Exp. Theor. Phys. Lett. 4 (1966) 160.

[9] J. Hirshfield and V. Granatstein, The Electron Cyclotron Maser - An Historical Survey, IEEE Trans. Microwave Theory Tech. 25 (1977) 522.

[10] J. Gardelle, L. Courtois, P. Modin and J. T. Donohue, Observation of coherent smith-purcell radiation using an initially continuous flat beam, Phys. Rev. ST Accel. Beams 12 (2009) 110701.

[11] C. Kittel and H. Kroemer, Thermal physics, $2^{\text {nd }}$ edition, WH Freeman, New York (1980).

[12] H.L. Andrews, C.A. Brau, J.D. Jarvis, C.F. Guertin, A.O’Donnell, B. Durant et al., Observation of THz evanescent waves in a smith-purcell free-electron laser, Phys. Rev. ST Accel. Beams 12 (2009) 080703. 